\title{
Deriving space-time variograms from space-time autoregressive (STAR) model specifications
}

\author{
Daniel A. Griffith ${ }^{1}$, Gerard B.M. Heuvelink ${ }^{2}$ \\ ${ }^{1}$ Ashbel Smith Professor, University of Texas at Dallas \\ Email: dagriffith@utdallas.edu \\ ${ }^{2}$ Wageningen University and Research Centre \\ Email: gerard.heuvelink@wur.nl
}

KEY WORDS: additive semi-variogram, autoregressive model, multiplicative semi-variogram, space, space-time, STAR, time

\begin{abstract}
:
Many geospatial science subdisciplines analyze variables that vary over both space and time. The space-time autoregressive (STAR) model is one specification formulated to describe such data. This paper summarizes STAR specifications that parallel geostatistical model specifications commonly used to describe space-time variation, with the goal of establishing synergies between these two modeling approaches. Resulting expressions for space-time correlograms derived from $1^{\text {st }}$-order STAR models are solved numerically, and then linked to appropriate space-time semivariogram models.
\end{abstract}

\section{Introduction}

Geostatistics furnishes techniques for modeling the covariance matrix, whereas spatial autoregression furnishes techniques for modeling the inverse covariance matrix, for a set of $n$ geographically distributed values of a single random function. Both seek to capture spatial autocorrelation effects in georeferenced data.

Although, in practice, both geostatistics and spatial autocorrelation techniques mostly are applied to static spatial variables, a growing interest among researchers is to utilize these techniques to address change over both space and time. Incorporating time is more than just adding another dimension, because the behavior of a variable over time differs from its behavior over space, and characteristics of temporal processes often are known to some degree. Accordingly, a space-time geostatistical or autoregressive model must capture the fundamental differences between spatial and temporal variation, and must include these differences in its structure and parameterization.

The purpose of this paper is to establish the basis for a synergy between space-time geostatistics and autoregressive (STAR) approaches to the modeling of correlation structures latent in space-time data. The mutually advantageous conjunction of these two approaches follows that established for the static case by Griffith and Csillag (1993) and Griffith and Layne (1997), and seeks to create an enhanced combined approach to the modeling of space-time correlation structures. Simple $1^{\text {st }}$ - and $2^{\text {nd }}$-order geographic neighbor direct dependency structures are addressed, with conceptualizations furnished by especially Gasim (1988) allowing them to be extended to larger neighborhoods. In doing so, we exploit the notion that a space-time semivariogram is valid (i.e., nonnegative definite) when any linear combination of values of the associated random function at any finite number of space-time points has non-negative variance.

\section{The Configurational Structure of Georeferenced Data}

Consider a variable $\mathrm{Z}=\{\mathrm{Z}(\mathrm{s}, \mathrm{t}) \mid \mathbf{s} \in \mathrm{S}, \mathrm{t} \in \mathrm{T}\}$ that varies within a spatial domain $S$ and a time horizon $T$. Let $Z$ be observed at $n$ space-time points $\left(\mathbf{s}_{\mathrm{i}}, \mathrm{t}_{\mathrm{j}}\right), \mathrm{i}=1,2, \ldots, \mathrm{m}$ and $\mathrm{j}=$ $1,2, \ldots, k$, where $n=m k$. These space-time observations constitute a time series of length $\mathrm{k}$ at each of the $\mathrm{m}$ spatial locations, and imply the use of a regular sampling scheme (i.e., the observations are uniformly spaced over time at each spatial location).

The set of $\mathrm{n}$ points can be converted to a surface partitioning by constructing its associated set of Thiessen (Voronoi) polygons; these become volumes in three dimensions. This conversion allow the generation of a Delauany triangulation (the dual graph) that furnishes a topology-based articulation of the configurational structure of the set of $n$ points. Interpoint distances furnish another. Suppose variable $\mathrm{Z}$ is an areal unit aggregate observed for $\mathrm{m}$ regions in time, where $\mathrm{k}$ is the frequency of observations per region over time. Let these areal units form a mutually exclusive and collectively exhaustive partitioning of a surface. If these polygons are convex hulls (all internal angles $<180^{\circ}$ ), then the geometric centroid of each polygon can be computed, and this set of points can be used both to convert the surface partitioning into a geographic distributions of points, and to construct a dual graph (similar to a Delaunay triangulation). This graph commonly is constructed using criteria based on chess piece movements: the rook's case (i.e., links connect points for polygons that share a common non-zero length boundary), and the queen's case (i.e., links connect points for polygons that share a common zero-i.e., point - or non-zero length boundary). For concave hulls (e.g., polygons with at least one internal angle $>180^{\circ}$ ) or for nested areal units (e.g., one contained completely inside another), a judiciously selected arbitrary point may be the dual graph node. Meanwhile, time can be represented with a simple line graph comprising a linear sequence of links and points.

In all three geographic cases, the graphs in question can be converted to adjacency matrices, $\mathbf{C}$, which are binary $0-1$ matrices with all diagonal entries being 0 . Because these graphs are planar or near-planar and connected, the number of ones in the m-by-m matrices representing geographic arrangement is at least $2(\mathrm{~m}-1)$, usually does not exceed $3(\mathrm{~m}$ 2 ), and never exceeds $8 \mathrm{~m}$. These matrices are symmetric here, in part because geographic dependencies are being cast as non-directional. The number of ones in the k-by-k time sequencing matrix is $2(\mathrm{k}-1)$. This set of matrices furnishes the building blocks of $n$-by-n space-time data matrices. Eigenfunctions extracted from each of these binary matrices can be used to summarize their respective structure. 


\section{STAR Model Specifications}

STAR model specifications (see Cliff et al. 1975) are explicit formulations describing how a variable $Z$ varies in space $\mathbf{s}=$ $(\mathrm{x}, \mathrm{y})$ and time $(\mathrm{t})$ in some joint fashion $(\mathrm{x}, \mathrm{y}, \mathrm{t})$. The following two linear discrete cases are of interest here:

$$
\begin{aligned}
& \mathrm{Z}(\mathrm{x}, \mathrm{y}, \mathrm{t})=\mathrm{a} \cdot \mathrm{Z}(\mathrm{x}, \mathrm{y}, \mathrm{t}-\Delta \mathrm{t})+\mathrm{b} \cdot\{\mathrm{Z}(\mathrm{x}-\Delta \mathrm{x}, \mathrm{y}, \mathrm{t}-\Delta \mathrm{t})+\mathrm{Z}(\mathrm{x}+\Delta \mathrm{x}, \\
& \mathrm{y}, \mathrm{t}-\Delta \mathrm{t})+\mathrm{Z}(\mathrm{x}, \mathrm{y}-\Delta \mathrm{y}, \mathrm{t}-\Delta \mathrm{t})+\mathrm{Z}(\mathrm{x}, \mathrm{y}+\Delta \mathrm{y}, \mathrm{t}-\Delta \mathrm{t})\}+\varepsilon(\mathrm{x}, \mathrm{y}, \mathrm{t}),
\end{aligned}
$$

and

$$
\begin{aligned}
& Z(x, y, t)=a \cdot Z(x, y, t-\Delta t)+b \cdot\{Z(x-\Delta x, y, t)+Z(x+\Delta x, y, \\
& t)+Z(x, y-\Delta y, t)+Z(x, y+\Delta y, t)\}+\varepsilon(x, y, t)
\end{aligned}
$$

Equation (1) specifies a value at location $(\mathrm{x}, \mathrm{y}, \mathrm{t})$ as a function of the preceding in situ value (time $t-\Delta t$ ) as well as the preceding neighboring values, a lagged specification. Equation (2) specifies a value at location $(\mathrm{x}, \mathrm{y}, \mathrm{t})$ as a function of the preceding in situ value (time $\mathrm{t}-\Delta \mathrm{t}$ ) as well as the contemporaneous neighboring values, a spatially contemporaneous specification. The random process $\varepsilon$ is white noise, which is uncorrelated in space and time. In the STAR model, correlation in space and time is captured by the autoregressive structure of the model (i.e., the response variable appears in both sides of the equations). Feedback loops or cycles make equation (2) fundamentally different from equation (1). An initial field for $t=0$ and spatial boundary conditions are needed in these formulations. In this paper, interest is in the case where sufficient time has transpired and the spatial extent is sufficiently large to allow negligible effects from boundary conditions.

\subsection{Theoretical Space-time Correlations}

Theoretical correlations can be posited for equations (1) and (2). Consider an infinite regular square (i.e., equal-sized pixels) tessellation lattice for which spatial adjacency (i.e., geographic neighbors) is defined by whether or not two square cells share a non-zero length common boundary (i.e., the rook's definition). Let $\{\mathrm{Z}(\mathbf{s})\}$ be a Gaussian random variable distributed across the vector of locations $\mathbf{s}$ (i.e., cells), such that $\{Z(\mathbf{s})\}$ and $\{Z(\mathbf{s}+\mathbf{h})\}$, for locations shifted by $\mathbf{h}$ units, are stochastically equivalent (i.e., complete stationarity). Spectral theory (Bartlett 1975; Haining 1978) reveals that the appropriate correlation function for the additive specification [i.e., equation (1)] is given by

$$
\rho_{\mathrm{h}, \mathrm{g}, \mathrm{k}}=\frac{\int_{0}^{\pi} \int_{0}^{\pi} \int_{0}^{\pi} \frac{\cos (\mathrm{h} \times \mathrm{t}) \cos (\mathrm{g} \times \mathrm{u}) \cos (\mathrm{k} \times \mathrm{v})}{\left\{1-\rho_{\mathrm{T}} \cos (\mathrm{t})-\rho_{\mathrm{s}} \cos (\mathrm{u})-\rho_{\mathrm{s}} \cos (\mathrm{v})\right\}^{\eta}} \mathrm{dtdudv}}{\int_{0}^{\pi} \int_{0}^{\pi} \int_{0}^{\pi} 1 /\left\{1-\rho_{\mathrm{T}} \cos (\mathrm{t})-\rho_{\mathrm{s}} \cos (\mathrm{u})-\rho_{\mathrm{s}} \cos (\mathrm{v})\right\}^{\eta} \mathrm{dt} \mathrm{dudv}},
$$

whereas that for the multiplicative specification [i.e., equation (2)] is given by $\rho_{\mathrm{h}, \mathrm{g}, \mathrm{k}}=\frac{\int_{0}^{\pi} \int_{0}^{\pi} \int_{0}^{\pi} \frac{\cos (\mathrm{h} \times \mathrm{t}) \cos (\mathrm{g} \times \mathrm{u}) \cos (\mathrm{k} \times \mathrm{v})}{\left[1-\cos (\mathrm{t})\left\{\rho_{\mathrm{s}}[\cos (\mathrm{u})+\cos (\mathrm{v})]+\rho_{\mathrm{T}}\right\}\right]^{\eta}} \mathrm{dt} d \mathrm{dv}}{\int_{0}^{\pi} \int_{0}^{\pi} \int_{0}^{\pi} 1 /\left[1-\cos (\mathrm{t})\left\{\rho_{\mathrm{s}}[\cos (\mathrm{u})+\cos (\mathrm{v})]+\rho_{\mathrm{T}}\right\}\right]^{\eta} \mathrm{dt} d \mathrm{udv}}$,

for temporal lag $\mathrm{h}(\mathrm{h}=0,1, \ldots)$, and spatial lags $\mathrm{g}(\mathrm{g}=0,1$, $\ldots)$ and $\mathrm{k}(\mathrm{k}=0,1, \ldots)$, where a positive integer value of $\eta$ yields a $\eta^{\text {th }}$-order model, $\rho_{\mathrm{s}}$ is the spatial and $\rho_{\mathrm{T}}$ is the temporal autoregressive parameter, and $(\mathrm{h}, \mathrm{g}, \mathrm{k})$ denotes the space-time lag involved.

\subsection{Space-time Autoregressive Structures}

The eigenvalues of the n-by-n connectivity matrix $\mathbf{C}$ for a linear surface partitioning containing $P$ cells are $2 \cos \left(\frac{p \pi}{P+1}\right), p=1,2, \ldots, P$. The 2 can be absorbed into the autoregressive parameter values, $\rho_{j}$, doubling the size of each feasible. This solution can be extended to two- and three-dimensional regular square lattice structures. Ord (1975) first reported the eigenvalues of the PQ-by-PQ connectivity matrix for a square tessellation surface partitioning forming a P-by-Q $(\mathrm{n}=\mathrm{PQ})$ complete rectangular region as $2\left[\cos \left(\frac{\mathrm{p} \pi}{\mathrm{P}+1}\right)+\cos \left(\frac{\mathrm{q} \pi}{\mathrm{Q}+1}\right)\right], \mathrm{p}=1,2, \ldots, \mathrm{P}$, and $\mathrm{q}=1,2, \ldots$, Q. Gasim (1988) presents extensions to Ord's results. And, Basilevsky (1983) summarizes the conventional time-series results. Here the three-dimensional matrix representation is given by

$$
\mathbf{C}=\mathbf{I}_{\mathrm{T}} \otimes \mathbf{I}_{\mathrm{s}}-\rho_{\mathrm{s}} \mathbf{I}_{\mathrm{T}} \otimes \mathbf{C}_{\mathrm{s}}-\rho_{\mathrm{T}} \mathbf{C}_{\mathrm{T}} \otimes \mathbf{I}_{\mathrm{s}},
$$

where $\otimes$ denotes Kronecker product, $\mathbf{I}_{\mathrm{T}}$ denotes the T-by-T identify matrix, $\mathbf{I}_{\mathrm{s}}$ denotes the PQ-by-PQ identity matrix, and $\mathbf{C}_{\mathrm{s}}=\mathbf{C}_{\mathrm{P}} \otimes \mathrm{I}_{\mathrm{Q}}+\mathbf{C}_{\mathrm{Q}} \otimes \mathbf{I}_{\mathrm{P}}$, for a P-by-Q rectangular square lattice, where $\mathbf{C}_{\mathrm{j}}$ is a matrix of $0 \mathrm{~s}$ except for the upper- and lower-off diagonals, which contain $1 \mathrm{~s}(\mathrm{j}=\mathrm{P}, \mathrm{Q}$, and $\mathrm{T}) . \mathrm{C}_{\mathrm{P}}$ and $\mathbf{C}_{\mathrm{Q}}$ have the same structure as $\mathbf{C}_{\mathrm{T}}$.

Equation (3) describes the correlogram values for spacetime data characterized by equation (1), whereas equation (4) describes space-time data characterized by equation (2). The three-dimensional connectivity matrix representation is given by

$$
\mathbf{C}=\mathbf{I}_{\mathrm{T}} \otimes \mathbf{I}_{\mathrm{s}}-\rho_{\mathrm{s}} \mathbf{C}_{\mathrm{T}} \otimes \mathbf{C}_{\mathrm{s}}-\rho_{\mathrm{T}} \mathbf{C}_{\mathrm{T}} \otimes \mathbf{I}_{\mathrm{s}},
$$

where $1-\cos (\mathrm{t})\left\{\rho_{\mathrm{s}}[\cos (\mathrm{u})+\cos (\mathrm{v})]+\rho_{\mathrm{T}}\right\}$ are the limiting eigenvalues of the space-time connectivity matrix C. Additional discussion of this topic appears in Griffith (1996).

Because the eigenvalues define the spectrum of a matrix, they appear in the denominator of equations (3) and (4); these denominators are based upon the limiting eigenvalues of the connectivity matrix representation of the space-time three-dimensional structure of data. In addition, Griffith and Csillag (1993) note, in contrast to the current thinking of that time, that a simultaneous autoregressive model can be por- 
trayed by letting $\eta=2$ in the denominator of equations (3) and (4)-it becomes a $2^{\text {nd }}$-order covariance specification; Bartlett (1975, pp. 19, 25) reports this result. Furthermore, Griffith and Layne (1997) summarize the close numerical connections between the spatial-only form of equations (3) and (4) and geostatistical semivariogram models.

\subsection{Space-time Covariance Functions in Geostatistics}

An important issue in the space-time geostatistical literature concerns whether or not the space and the time components of a formulated function are: separable such that they factor (Gneiting et al., 2006); or, nonseparable such that they form a linear combination (Ma 2008). Mitchell et al. (2005) propose a modified multivariate repeated measures likelihood ratio test coupled with bootstrapping for this purpose. Brown et al. (2001) note that separability requires that the expected value for some random variable at location $(x, y)$ in time $t+1$, given its values in a neighborhood of location $(x, y)$ in time $t$, must equal the conditional expectation just for location ( $\mathrm{x}$, y).

Stein (2005) furnishes an overview of space-time covariance and aspects of spatial-temporal interaction, and proposes a new class of space-time covariances. Ma (2003, 2008) presents methods for constructing spatio-temporal stationary covariance models, and supplements the set presented by Kolovos et al. (2004). Gneiting et al. (2006) posit theorems for symmetric and separable specifications, the Cressie-Huang and the Gneiting model, and stationarity. Fuentes et al. (2008) propose a nonstationary and nonseparable spectral density specification for which separability is a special case. Finally, Calder (2007) proposes a Bayesian specification that includes priors on initial points in time.

The space-time separability assumption (Bogaert 1996) states that the space-time covariance function $\mathrm{C}(\mathrm{h}, \mathrm{u})$ can be written as a product of a spatial, $\mathrm{C}_{\mathrm{S}}(\mathrm{h})$, and a temporal, $\mathrm{C}_{\mathrm{T}}(\mathrm{u})$, covariance function, such that

$$
\mathrm{C}(\mathrm{h}, \mathrm{u})=\mathrm{C}_{\mathrm{S}}(\mathrm{h}) \cdot \mathrm{C}_{\mathrm{T}}(\mathrm{u}) .
$$

One non-separable specification expresses the space-time covariance function as a linear combination of these two components (De Cesare et al. 2001), such that

$$
\mathrm{C}(\mathrm{h}, \mathrm{u})=\mathrm{C}_{\mathrm{S}}(\mathrm{h})+\mathrm{C}_{\mathrm{T}}(\mathrm{u})+\mathrm{p} \mathrm{C}_{\mathrm{S}}(\mathrm{h}) \cdot \mathrm{C}_{\mathrm{T}}(\mathrm{u}),
$$

which is statistically valid if both $\mathrm{C}_{\mathrm{S}}(\mathrm{h})$ and $\mathrm{C}_{\mathrm{T}}(\mathrm{u})$ are valid covariance functions and parameter $p$ satisfies certain conditions (De Cesare et al. 2001). This product-sum model appears to perform well in practice (De Iaco et al. 2003, Gething et al. 2007).

Another alternative is the metric model (Dimitrakopoulos and Luo 1994), which reduces the space-time covariance function to

$$
\mathrm{C}(\mathrm{h}, \mathrm{u})=\mathrm{C}_{\mathrm{ST}}\left(\sqrt{\mathrm{h}^{2}+(\alpha \cdot \mathrm{u})^{2}}\right)
$$

whose essential characteristic is that distance in space is made comparable to distance in time through the scaling parameter $\alpha$. Equation (7) is rather restrictive because it assumes that the variances in time and space are equal. The following more flexible specification results from combining equation (6) with $\mathrm{p}=0$ and equation (7):

$$
\mathrm{C}(\mathrm{h}, \mathrm{u})=\mathrm{C}_{\mathrm{S}}(\mathrm{h})+\mathrm{C}_{\mathrm{T}}(\mathrm{u})+\mathrm{C}_{\mathrm{ST}}\left(\sqrt{\mathrm{h}^{2}+(\alpha \cdot \mathrm{u})^{2}}\right) .
$$

The third term in the right-hand side represents a joint spacetime interaction effect.

\section{Numerical Experiments}

Only numerical integration solutions to the definite integrals in equations (3) and (4) are available here. Because this integration is numerical intensive, and $1^{\text {st }}$ - and $2^{\text {nd }}$-order results are similar, only $1^{\text {st }}$-order models are assessed. Because the exponent in the denominators of the integrands is 1 , equations (1) and (2) refer to a space-time conditional autoregressive (CAR) specification. Numerical results for equations (3) and (4) were calculated for time lags $h=0,1$, $\ldots, 65$ and space lags $g$ and $\mathrm{k}=0,1, \ldots, 50$, using the autoregressive parameter pairs $\left\{\left(\rho_{\mathrm{s}}, \rho_{\mathrm{T}}\right):(0.49,0.01),(0.40\right.$, $0.19),(0.30,0.39),(0.20,0.59),(0.10,0.79),(0.01,0.97)\}$ (see Table 1). Theoretical nugget and sill values for equations (5)-(8) respectively are 0 and 1 . Deviations from these values represent specification error; the numerical integration error is negligible.

The stable, the Bessel, and the exponential variogram models were evaluated in terms of their fits to these numerical data, with the exponential variogram model performing the best. Estimation results for this model appear in Tables 1 and 2, and suggest that equation (5) does not furnish a good description of the space-time structure generated by equations (1) and (2). Equation (8) fails to provide any improvement in the description furnished by equation (7), because equations (1) and (2) generate realizations from a random function that have the same sill (variance) in time and space; in cases where the variances differ, equation (8) will almost surely do better than equation (7). Equation (7) appears to yield a marginally better description than the one provided by equation (6). The principal difference between the relationship between equation (7) and equations (1) and (2) is the estimated $\alpha$ parameter, the anisotropic weight attached to the time distance in order to differentiate it from space distance, which is included in the specification of equation (7), but not equations (1) and (2).

\section{Conclusions}

In summary, numerical evaluation suggests that the STAR model equations (1) and (2) yield the metric model equation (7) with exponential-shaped variograms. However, realworld processes may, in addition to the space-time models characterized by equations (1) and (2), have purely spatial and purely temporal components. Whittle (1954) shows that purely spatial AR models have Bessel function-shaped covariance functions, whereas linear one-dimensional time series models have exponential variograms. Thus, processes that also have purely temporal and/or spatial components should be characterized by variogram models given by equa- 
tion (8) rather than equation (7). Assuming that the generating 


\begin{tabular}{|c|c|c|c|c|c|c|c|c|c|c|c|c|}
\hline \multirow{2}{*}{$\rho_{\mathrm{s}}$} & \multirow{2}{*}{$\rho_{\mathrm{T}}$} & \multicolumn{3}{|c|}{ space } & \multicolumn{3}{|c|}{ time } & \multirow{2}{*}{$\mathrm{a}$} & \multirow{2}{*}{ RESS } & \multicolumn{3}{|c|}{ space-time } \\
\hline & & $\mathrm{C} 0$ & $\mathrm{C} 1$ & $\mathrm{r}$ & $\mathrm{C} 0$ & $\mathrm{C} 1$ & $\mathrm{r}$ & & & $\mathrm{C} 0$ & $\mathrm{C} 1$ & $\mathrm{r}$ \\
\hline \multicolumn{13}{|c|}{ Equation (5) } \\
\hline 0.01 & 0.97 & 1.2052 & 0.0992 & 0.5212 & 0.7661 & 0.0006 & 4.9569 & & 0.8594 & & & \\
\hline 0.10 & 0.79 & 1.2315 & 0.0514 & 0.7712 & 0.7788 & 0.0007 & 2.7328 & & 0.9279 & & & \\
\hline 0.20 & 0.59 & 1.3987 & 0.0459 & 0.8321 & 0.6917 & 0.0006 & 2.4328 & & 0.9451 & & & \\
\hline 0.30 & 0.39 & 1.1825 & 0.1004 & 0.0000 & 0.7787 & 0.0008 & 1.9168 & & 0.9986 & & & \\
\hline 0.40 & 0.19 & 1.2671 & 0.0309 & 0.8431 & 0.7697 & 0.0007 & 1.6549 & & 0.9620 & & & \\
\hline 0.49 & 0.01 & 1.3535 & 0.0283 & 0.8041 & 0.7231 & 0.0006 & 1.3169 & & 0.9721 & & & \\
\hline \multicolumn{13}{|c|}{ Equation (6) } \\
\hline 0.01 & 0.97 & 0.0000 & 1.0000 & 0.4543 & 0.0096 & 0.9892 & 4.4235 & -1.0000 & 0.0054 & & & \\
\hline 0.10 & 0.79 & 0.0008 & 0.9992 & 0.5960 & 0.0281 & 0.9685 & 1.9877 & -1.0000 & 0.0441 & & & \\
\hline 0.20 & 0.59 & 0.0000 & 1.0000 & 0.5964 & 0.0270 & 0.9691 & 1.3773 & -1.0000 & 0.0684 & & & \\
\hline 0.30 & 0.39 & 0.0105 & 0.9894 & 0.5675 & 0.0106 & 0.9852 & 1.0190 & -1.0000 & 0.0739 & & & \\
\hline 0.40 & 0.19 & 0.0079 & 0.9920 & 0.5076 & 0.0049 & 0.9907 & 0.7222 & -1.0000 & 0.0673 & & & \\
\hline 0.49 & 0.01 & 0.0006 & 0.9992 & 0.2962 & 0.0000 & 0.9952 & 0.3038 & -1.0000 & 0.1187 & & & \\
\hline \multicolumn{13}{|c|}{ Equation (7) } \\
\hline 0.01 & 0.97 & & & & & & & 0.0078 & 0.0029 & 0.0052 & 0.9948 & 0.3869 \\
\hline 0.10 & 0.79 & & & & & & & 0.0714 & 0.0340 & 0.0208 & 0.9792 & 0.5214 \\
\hline 0.20 & 0.59 & & & & & & & 0.1585 & 0.0560 & 0.0207 & 0.9793 & 0.5414 \\
\hline 0.30 & 0.39 & & & & & & & 0.2784 & 0.0601 & 0.0174 & 0.9826 & 0.5381 \\
\hline 0.40 & 0.19 & & & & & & & 0.4727 & 0.0526 & 0.0126 & 0.9874 & 0.5145 \\
\hline 0.49 & 0.01 & & & & & & & 0.8494 & 0.1137 & 0.0044 & 0.9956 & 0.8494 \\
\hline \multicolumn{13}{|c|}{ Equation (8) $^{\mathrm{a}}$} \\
\hline 0.01 & 0.97 & 0 & $* * *$ & $* * *$ & 0 & 0.0014 & $* * *$ & 0.0792 & 0.0029 & 0 & 0.9986 & 0.3869 \\
\hline 0.10 & 0.79 & 0 & 0.0001 & 32.9987 & 0 & 0.0029 & 1.9357 & 0.0737 & 0.0337 & 0 & 0.9970 & 0.5135 \\
\hline 0.20 & 0.59 & 0 & 0.0001 & 16.0388 & 0 & 0.0034 & 2.1593 & 0.1635 & 0.0552 & 0 & 0.9966 & 0.5317 \\
\hline 0.30 & 0.39 & 0 & 0.0001 & 13.7028 & 0 & 0.0035 & 2.2861 & 0.2857 & 0.0588 & 0 & 0.9964 & 0.5280 \\
\hline 0.40 & 0.19 & 0 & 0.0001 & 10.1898 & 0 & 0.0037 & 2.2348 & 0.4825 & 0.0509 & 0 & 0.9963 & 0.5046 \\
\hline 0.49 & 0.01 & 0 & 0.0002 & 7.2160 & 0 & 0.0044 & 2.1385 & 0.8653 & 0.1112 & 0 & 0.9954 & 0.3806 \\
\hline
\end{tabular}

processes satisfies the linear ARMA model, the temporal and spatio-temporal variograms may be described with exponential functions, whereas the spatial component may be described with a Bessel function.

\section{REFERENCES}

Bartlett, M. (1975). The Statistical Analysis of Spatial Pattern. London, Chapman-Hall.

Basilevsky, A. (1983). Applied Matrix Algebra in the Statistical Sciences. NY, North-Holland.

Bogaert, P. (1996) "Comparison of kriging techniques in a space-time context." Mathematical Geology 28, 7386.

Brown, P., P. Diggle, M. Lord, and P. Young. (2001).

"Space-time calibration of radar rainfall data." Applied Statistics 50, 221-241.

Calder, C. (2007). "Dynamic factor process convolution models for multivariate space-time data with application to air quality assessment." Environmental and Ecological Statistics 14, 229-247.

Cliff, A., P. Haggett, J. Ord, K. Bassett, and R. Davies. (1975). Elements of Spatial Structure. Cambridge, Cambridge U. Press.

De Cesare, L., D.E. Myers and D. Posa. (2001). "Product-sum covariance for space-time modeling: an environmental application." Environmetrics 12, 11-23.

De Iaco, S., D.E. Myers and D. Posa. (2003). "The linear coregionalization model and the product-sum spacetime variogram." Mathematical Geology 35, 25-38.

Dimitrakopoulos, R. and X. Luo. (1994). Spatiotemporal Modeling: Covariances and Ordinary Kriging Systems
Geostatistics for the Next Century. R. Dimitrakopoulos. Dordrecht, Kluwer. 88-93.

Fuentes, M., L. Chen, and J. Davis. (2008). "A class of nonseparable and nonstationary spatial temporal covariance functions." Environmetrics 19, 487-507.

Gasim, A. (1988). "First-order autoregressive models: a method for obtaining eigenvalues for weighting matrices." J. of Statistical Planning and Inference 18, 391398.

Gneiting, T., M. Genton, and P. Guttorp. (2006). Chapter 4 - Statistical Methods of Spatio-Temporal Systems. B. Finkenstaedt, L. Held, and V. Isham. Boca Raton, Chapman-Hall. 151-175.

Gething, P.W., P.M. Atkinson, A.M. Noor, P.W. Gikandi, S.I. Hay and M.S. Nixon. (2007). "A local space-time kriging approach applied to a national outpatient malaria data set." Computers \& Geosciences 33, 1337-1350.

Griffith, D. (1996). Spatial Statistical Analysis and GIS: Exploring Computational Simplifications for Estimating the Neighborhood Spatial Forecasting Model Spatial Analysis: Modelling in a GIS Environment. P. Longley and M. Batty. London, Longman GeoInformation. 255-268.

Griffith, D., and F. Csillag. (1993). "Exploring relationships between semi-variogram and spatial autoregressive models." Papers in Regional Science 72, 283295.

Griffith, D., and L. Layne. (1997). Uncovering Relationships Between Geo-Statistical and Spatial Autoregressive Models - 1996 Proceedings on the Section on Statistics and the Environment. Washington, D.C., American Statistical Association. 91-96. 
Haining, R. (1978). Specification and Estimation Problems in Models of Spatial Dependence. Evanston, IL, Department of Geography, Northwestern University.

Kolovos, A., G. Christakos, D. Hristopulos, and M. Serre. (2004). Methods for generating non-separable spatiotemporal covariance models with potential environmental applications." Advances in Water Resources $27,815-830$

Ma, C. (2003). "Families of spatio-temporal stationary covariance models." Journal of Statistical Planning and Inference 116, 489-501.

Ma, C. (2008). (2008). "Recent developments on the construction of spatio-temporal covariance models."
Stochastic Environmental Research and Risk Assessment 22, S39-S47.

Mitchell, M., M. Genton, and M. Gumpertz. (2005). Testing for separability of space-time covariances." Environmetrics 16, 819-831.

Ord, J. (1975). "Estimation methods for models of spatial interaction." Journal of the American Statistical Association 70, 120-126.

Stein, M. (2005). "Space-time covariance functions." Journal of the American Statistical Association 100, 310-321.

Whittle, P. (1954). "On Stationary Processes in the Plane.” Biometrika 41, 434-449.

\begin{tabular}{|c|c|c|c|c|c|c|c|c|c|c|c|c|}
\hline \multirow{2}{*}{$\rho_{\mathrm{s}}$} & \multirow{2}{*}{$\rho_{\mathrm{T}}$} & \multicolumn{3}{|c|}{ space } & \multicolumn{3}{|c|}{ time } & \multirow{2}{*}{$\mathrm{a}$} & \multirow{2}{*}{ RESS } & \multicolumn{3}{|c|}{ space-time } \\
\hline & & C0 & $\mathrm{C} 1$ & $\mathrm{r}$ & C0 & $\mathrm{C} 1$ & $\mathrm{r}$ & & & C0 & C1 & $\mathrm{r}$ \\
\hline \multicolumn{13}{|c|}{ Equation (5) } \\
\hline 0.01 & 0.97 & 1.2950 & 0.1065 & 0.5216 & 0.7130 & 0.0006 & 4.9405 & & 0.8597 & & & \\
\hline 0.10 & 0.79 & 1.3720 & 0.0563 & 0.7786 & 0.6995 & 0.0007 & 3.4037 & & 0.9366 & & & \\
\hline 0.20 & 0.59 & 1.3902 & 0.0440 & 0.8213 & 0.6966 & 0.0007 & 2.0341 & & 0.9525 & & & \\
\hline 0.30 & 0.39 & 1.3016 & 0.0337 & 0.9219 & 0.7481 & 0.0009 & 1.5630 & & 0.9607 & & & \\
\hline 0.40 & 0.19 & 1.3459 & 0.0292 & 0.9110 & 0.7263 & 0.0009 & 1.1788 & & 0.9711 & & & \\
\hline 0.49 & 0.01 & 1.2671 & 0.0210 & 1.0209 & 0.7750 & 0.0015 & 0.4744 & & 0.9816 & & & \\
\hline \multicolumn{13}{|c|}{ Equation (6) } \\
\hline 0.01 & 0.97 & 0.0000 & 1.0000 & 0.4564 & 0.0092 & 0.9897 & 4.4207 & -1.0000 & 0.0054 & & & \\
\hline 0.10 & 0.79 & 0.0003 & 0.9996 & 0.6219 & 0.0240 & 0.9733 & 1.9716 & -1.0000 & 0.1266 & & & \\
\hline 0.20 & 0.59 & 0.0000 & 0.9999 & 0.6627 & 0.1955 & 0.9779 & 1.3631 & -1.0000 & 0.1093 & & & \\
\hline 0.30 & 0.39 & 0.0000 & 0.9999 & 0.6925 & 0.0122 & 0.9856 & 1.0026 & -1.0000 & 0.0650 & & & \\
\hline 0.40 & 0.19 & 0.0039 & 0.9961 & 0.7478 & 0.0000 & 0.9986 & 0.7322 & -1.0000 & 0.0874 & & & \\
\hline 0.49 & 0.01 & 0.0000 & 0.9663 & 0.7875 & 0.8039 & 0.1919 & 0.2914 & -0.9999 & 0.7325 & & & \\
\hline \multicolumn{13}{|c|}{ Equation (7) } \\
\hline 0.01 & 0.97 & & & & & & & 0.0079 & 0.0030 & 0.0047 & 0.9953 & 0.3880 \\
\hline 0.10 & 0.79 & & & & & & & 0.0788 & 0.1190 & 0.0147 & 0.9853 & 0.5386 \\
\hline 0.20 & 0.59 & & & & & & & 0.2001 & 0.1008 & 0.0104 & 0.9896 & 0.5885 \\
\hline 0.30 & 0.39 & & & & & & & 0.4346 & 0.0575 & 0.0044 & 0.9955 & 0.6321 \\
\hline 0.40 & 0.19 & & & & & & & 1.0732 & 0.0826 & 0.0000 & 1.0000 & 0.7076 \\
\hline 0.49 & 0.01 & & & & & & & 10.3725 & 0.1179 & 0.0000 & 1.0000 & 0.9785 \\
\hline \multicolumn{13}{|c|}{ Equation (8) $^{\mathrm{a}}$} \\
\hline 0.01 & 0.97 & 0 & $* * *$ & $* * *$ & 0 & 0.0014 & $* * *$ & 0.0080 & 0.0030 & 0 & 0.9986 & 0.3880 \\
\hline 0.10 & 0.79 & 0 & 0.0001 & 18.4139 & 0 & 0.0019 & 3.2382 & 0.0802 & 0.1187 & 0 & 0.9981 & 0.5319 \\
\hline 0.20 & 0.59 & 0 & 0.0001 & 19.6642 & 0 & 0.0017 & 2.6567 & 0.2029 & 0.1005 & 0 & 0.9982 & 0.5830 \\
\hline 0.30 & 0.39 & 0 & 0.0001 & 8.1486 & 0 & 0.0019 & 2.9423 & 0.4382 & 0.0570 & 0 & 0.9981 & 0.6274 \\
\hline 0.40 & 0.19 & 0 & 0.0001 & 7.6018 & 0 & 0.0010 & 3.6334 & 1.0772 & 0.0824 & 0 & 0.9989 & 0.7054 \\
\hline 0.49 & 0.01 & 0 & 0.0003 & 0.7745 & 0 & 0.0002 & 7.8400 & 10.3960 & 0.1178 & 0 & 0.9996 & 0.9770 \\
\hline
\end{tabular}

